\title{
Efectos de la certificación de calidad ISO 9001:2008 en la gestión de los colegios de La Presentación de la provincia de Bogotá"
}

\section{Effects of the quality certification ISO 9001:2008 in the management of the colegios de La Presentación in the Bogotás provincia}

Recibido: 22 de enero de 2015 Revisado: 17 de febrero de 2015

Aceptado: 27 de junio de 2015

Sandra Liliana Becerra Londoño**

Icontec Internacional

Gloria Patricia Corredor Mendoza****

Colegio de La Presentación Ubaté

\section{RESUMEN}

Este artículo presenta los resultados de una investigación que determina los efectos que el sistema de gestión de la calidad, certificado con la norma técnica NTC ISO 9001:2008, ha generado en la gestión de seis colegios de La Presentación en un lapso de seis años. Teóricamente se retoman postulados del Ministerio de Educación Nacional de Colombia y de Elsa Castañeda (2010), en relación con la calidad en la educación y la certificación. La metodología incluye la definición de categorías para el análisis y cruce de variables.

* Artículo de investigación.

** Trabajadora Social. Consultora, auditora y docente Universitaria. Especialista en Administración y Gerencia de Sistemas de la Calidad. Magíster en Calidad y Gestión Integral. Correo electrónico salibelo21@gmail.com

*** Licenciada en Ciencias Religiosas. Pedagoga. Rectora del Colegio de la Presentación de Ubaté. Especialista en Administración y Gerencia de Sistemas de la Calidad. Magíster en Calidad y Gestión Integral. Correo electrónico gloriacorredor_924@hotmail.com 
En el alcance se incorporan los factores de eficiencia económica, los niveles de conocimiento, de aceptación y de resistencia en la comunidad; los beneficios y las estrategias de mejoramiento continuo para el direccionamiento estratégico.

Entre los efectos identificados se encuentran altos niveles de aceptación y conocimiento del sistema de gestión de la calidad, bajo nivel de resistencia relacionado con el trámite de la documentación; beneficios tangibles que redundan en la prestación del servicio educativo y factores de eficiencia económica del sistema reflejados en la sostenibilidad, la inversión, el enfoque al cliente, la toma de decisiones basada en hechos y una dinámica que integra la educación y la calidad.

Para la comunidad religiosa reviste importancia, ya que establece resultados concretos que reflejan el estado del arte en el proceso y sirven como elementos de enriquecimiento conceptual para futuras investigaciones en las áreas de la calidad y la educación.

Palabras clave: calidad y educación, certificación, gestión de la calidad, ISO 9001.

\section{ABSTRACT}

This article presents the results of an investigation that determines the effects that the quality management system, it was certified with the technical standard NTC ISO 9001: 2008, has generated in the management of six schools of La Presentation, within six years. Postulates of the Ministerio de Educación de Colombia and Elsa Castañeda (2010) are taken up in the theory in relation to the quality of education and certification. The methodology includes the definition of categories for analysis and cross variables.
In the scope, economic efficiency factors, levels of knowledge, acceptance and resistance in the community, benefits and continuous improvement strategies for the strategic direction, are incorporated.

Among the identified effects are high levels of acceptance and knowledge of quality management system, low resistance related to the processing of documentation; tangible benefits in the provision of educational services and factors of economic efficiency of the system reflected in sustainability, investment, customer focus, decision-making based on facts and a dynamic that integrates education and quality.

Keywords: Effects, quality and education, certification, quality management, ISO 9001.

\section{INTRODUCCIÓN}

La necesidad de establecer los efectos que la certificación de calidad ISO 9001:2008 genera en los colegios de La Presentación de la provincia de Bogotá — compuesta por las sedes de Ubaté, Zipaquirá, Sogamoso, Duitama, y Ferias y Centro en Bogotá- surge en un momento coyuntural en el que, habiendo transcurrido seis (6) años de la certificación de calidad, las directivas se cuestionan acerca de los resultados tangibles que dicha certificación deja en beneficio de los colegios, en la comunidad educativa y en la eficiencia económica en materia de sostenibilidad.

En ese sentido, la investigación responde a interrogantes tales como los siguientes: ¿qué factores de eficiencia económica se derivaron de la inversión realizada en la vigencia de la certificación y su relación con los principios de la calidad frente al retorno de la inversión? ¿Qué niveles de aceptación, conocimiento o resistencia tiene el sistema de gestión de la calidad vigente desde hace 6 años para las comunidades académicas de 
la provincia de Bogotá? ¿Qué beneficios identificaron los gestores y líderes de los procesos con la implementación, certificación y recertificación del sistema de gestión de la calidad en los colegios? ¿Qué estrategias de mejora continua se podrían proponer para potencializar el direccionamiento estratégico, conforme a los resultados obtenidos en torno a la calidad?

Para responder a estos interrogantes, la investigación plantea un acercamiento a las partes interesadas que conforman la comunidad educativa, especialmente a los padres de familia, los docentes, los estudiantes, el personal de apoyo, el administrativo y la comunidad religiosa, a partir de la aplicación de encuestas, entrevistas semiestructuradas, el diligenciamiento de formatos predeterminados y la interacción con dichos actores.

En el plano teórico, la investigación realiza un acercamiento a la comunidad religiosa y a su modelo pedagógico, a los planteamientos de la educación en el contexto Latinoamericano (Castañeda, 2010; UNESCO, 2002), en los que la educación es un concepto dinámico que varía de acuerdo con circunstancias históricas y sociales que tienen que ver con una serie de elementos culturales y teóricos que avanzan hacia enfoques de calidad, eficacia, relevancia individual y calidad de los procesos, y a los postulados del Ministerio de Educación Nacional en materia de calidad de la educación y su conjugación con los sistemas de gestión, la norma técnica ISO 9001: 2008 sobre gestión de la calidad y la Guía técnica de calidad 200:2011 para la aplicación de la ISO 9001 en establecimientos de educación, los antecedentes en investigaciones previas, así como las normas y leyes que rigen la educación y la gestión de la calidad, aportando los soportes para su desarrollo.

La investigación parte de tres hipótesis, en las que se afirma 1) el sistema de gestión de la calidad certificado desde 2007 mejora la calidad del servicio educativo que prestan los colegios de La Presentación de la provincia de Bogotá; 2) el sistema de gestión de la calidad en los colegios de La Presentación de la provincia de Bogotá ha tenido mayores niveles de aceptación que de resistencia desde que se certificaron en 2007; y 3) el sistema de gestión de la calidad genera un mayor desgaste administrativo en relación con los beneficios obtenidos en el servicio educativo y no se sostiene económicamente en relación con los presupuestos asignados.

El aporte de la investigación se ubica en los postulados y en las conclusiones para las instituciones educativas de la Congregación de Hermanas de la Caridad Dominicas de La Presentación de la Santísima Virgen, y para aquellas otras instituciones de educación básica secundaria y media vocacional, con el fin de que sean considerados como marco de referencia para la toma de decisiones o para el planteamiento de investigaciones futuras que pretendan responder a otros interrogantes relativos a la gestión de la calidad. Esto le proporciona a la investigación un carácter aplicado el que sus principales destinatarios son los directivos y los actores sociales de la educación en instituciones similares.

Lo anterior reviste importancia para la provincia de Bogotá, ya que los resultados de la investigación, además, se constituyen en un instrumento para la toma de decisiones acerca de la terminación, continuidad o posible expansión hacia la implementación de otros sistemas de gestión. Esto permite realizar un análisis a la luz de cuatro grandes campos que combinan elementos cualitativos y cuantitativos, a saber: primero, un análisis de la eficiencia económica que observa si el sistema de gestión de la calidad certificado es sostenible, la redundancia en beneficios directos e indirectos para la educación que prestan los colegios y un estado del arte de la relación entre la eficiencia económica y los principios de la calidad; segundo, establece los niveles de aceptación, conocimiento y resistencia que el sistema de gestión de la calidad certificado ha dejado 
en la comunidad educativa, respecto de sus actores y del aporte a la calidad de la educación; tercero, determina de primera mano, con los actores involucrados en el proceso, los beneficios que el sistema ha dejado en su proceso de implementación, certificación y recertificación; finalmente, propone estrategias de mejora continua para potencializar el direccionamiento estratégico, dinamizar la gestión administrativa y el horizonte institucional en materia de la gestión de la calidad.

Es importante destacar que no se encuentran estudios conocidos acerca del establecimiento de efectos de un sistema de gestión de la calidad certificado en colegios, sin embargo, un estudio cercano de medición de impacto de la certificación de calidad en la misión y visión institucional, realizado por estudiantes de la Facultad de Educación de la Universidad Javeriana, concluye que "después de la certificación, muchos colegios tuvieron una mejor estructura curricular y se logró aclarar de mejor manera la propuesta formativa desde los planes de estudio y el PEI. Tanto padres de familia como estudiantes reconocen mayor énfasis en la formación de los valores que la institución posee dentro de su marco visional y que los estudiantes han ido apropiando de manera más fuerte que antes de la certificación" (Aristizábal et al, 2009).

\section{MÉTODO}

La metodología parte del planteamiento de un estudio explicativo que da respuesta a los interrogantes planteados, con carácter aplicado (Medina, 2007, p. 55 ), en la medida en que los resultados se constituyen en fuente para intervenir la realidad de los colegios objeto de estudio. La investigación desarrolla un enfoque mixto, ya que no solo pretende hacer hincapié en la explicación por medio del análisis de datos, sino que logra una perspectiva más amplia y profunda e involucra la observación y la interacción directa con los gestores del sistema de gestión de la calidad en los colegios de La Presentación. La búsqueda se divide en cuatro momentos: en el primero, el lógico (Universidad Nacional de Colombia, s. f.), se desarrolla toda la formulación del problema y, en general, del proceso metodológico; el segundo permite analizar los procedimientos, las técnicas y los instrumentos utilizados en la recolección de datos; el tercero aborda formas y procedimientos que permiten recolectar y organizar la información, aplicando para ello cada uno de los instrumentos; y finalmente se realizan la elaboración teórica y el análisis definitivo de los datos recolectados, conforme a los criterios de análisis de la información.

Las técnicas seleccionadas fueron la encuesta, la entrevista semiestructurada y el diligenciamiento de una matriz de eficiencia económica. Para la encuesta se tomó una muestra de carácter aleatorio equivalente al $9.1 \%$ de la población, con un margen de error del $3 \%$ y un nivel de confianza del $96 \%$, que dio como resultado 1010 integrantes de la comunidad educativa. La entrevista semiestructurada se realizó a dos miembros de la comunidad educativa, líderes de proceso y gestores del sistema de gestión de calidad, y la matriz de eficiencia económica se aplicó en los economatos de cada colegio, para determinar la inversión, los costos directos e indirectos en términos financieros y la calificación de aspectos relacionados con los principios de la calidad frente al retorno de la inversión.

Para la selección de las muestras se tuvieron en cuenta los siguientes criterios: la antigüedad de los miembros, su participación en el proceso de la calidad y la rigurosidad de respuesta en el caso de los datos financieros; así mismo, por la necesidad de interacción con la comunidad educativa, los instrumentos se aplicaron de manera presencial. 
Para el análisis e interpretación de la información, los resultados se clasificaron pasando de una escala numérica a una ordinal, que determinó los niveles esperados y las categorías centrales relacionadas con la eficiencia económica, la aceptación, la resistencia y el conocimiento del sistema de gestión de la calidad, así como los beneficios legados de este.

En el mismo sentido, se crearon como categorías para el análisis de datos y para establecer resultados concretos, las siguientes: costos directos, costos indirectos, asignación presupuestal en términos de inversión, sostenibilidad, incrementos de los costos educativos, principios de la calidad frente al retorno de la inversión, como parte de la variable de eficiencia económica; padres de familia y estudiantes, docentes, personal administrativo y religioso, como parte de la variable de conocimiento; resistencia y aceptación; y, finalmente, frente a la variable de beneficios, la implementación, certificación y madurez del sistema de gestión de la calidad. El cruce de dichas variables se tradujo en el establecimiento de los efectos del sistema.

Para cada instrumento de recolección de datos (entrevistas, encuestas, revisión documental y observación directa), se desarrolló una metodología de análisis que incluye la estadística descriptiva, la codificación de información con análisis de frecuencias y el análisis de información financiera, en conjugación con los elementos cualitativos. Finalmente, un análisis global permitió la combinación de los aspectos cualitativos y cuantitativos, así como la confrontación de las hipótesis en términos de falso o verdadero.

\section{RESULTADOS}

La información recolectada y procesada en los colegios de La Presentación, mediante la aplicación de los métodos descritos anteriormente, permitió consolidar los efectos que, a la luz de los temas de estudio, plasmados en los objetivos, reflejan la realidad de los colegios de La Presentación en la provincia de Bogotá. Entendiendo que un efecto puede ser la desviación de lo que se espera - bien sea positivo, negativo 0 ambos

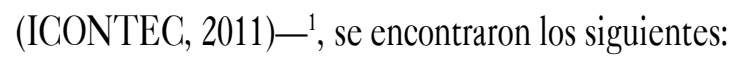

\section{Factores de eficiencia económica}

Fueron categorizados en el incremento de los costos educativos y en la variación de ingresos de acuerdo con dichos costos. El ingreso de las instituciones educativas se incrementa debido al mayor valor permitido en el cobro de costos educativos, al estar los colegios certificados en gestión de la calidad.

En cuanto a costos directos, es decir, los relacionados con los pagos al ente certificador y la capacitación para el mantenimiento del sistema —con excepción del año 2010-, los ingresos por certificación han sostenido este egreso, dando un margen superior al cincuenta por ciento de utilidad. En relación con la sostenibilidad, el ingreso adicional para los colegios certificados sostiene los costos directos relacionados con el presupuesto para el sistema y se conjuga con la inversión que fortalece la gestión y el quehacer misional.

La mejora producto de la retroalimentación del cliente, el aumento de la competitividad, la mejora de la sostenibilidad, el liderazgo, la participación del personal, la gestión por procesos y el enfoque del sistema para la gestión se ubican en un nivel de calificación alto frente al retorno de la inversión. En términos de eficiencia económica, la mejora presenta un nivel de desempeño medio en reducción de costos, mejora de la rentabilidad, mejora de los ingresos, mejora del desempeño del presupuesto y mejora del retorno de la inversión. El desempeño de los proveedores muestra

1 Definición de riesgo, nota 1. 
un valor agregado en la relación con la constancia, la fidelidad y el compromiso.

\section{Apropiación}

El conocimiento del sistema de gestión de la calidad por parte de los líderes de los procesos es amplio. Se destaca el dominio por parte de los docentes, el personal de apoyo, el personal administrativo y la comunidad religiosa. No obstante, tanto los estudiantes como los padres de familia conocen el sistema de gestión de la calidad en un nivel medio alto.

La aceptación del sistema y la satisfacción frente a la prestación del servicio son predominantes entre las partes de la comunidad educativa y consideran que la certificación del sistema de gestión de la calidad ha traído beneficios y es aceptado por la comunidad educativa de los colegios de la provincia.

Los niveles de resistencia se encuentran en un nivel bajo. Sin embargo, los estudiantes consideran que los trabajos en el aula podrían ser más sencillos, los docentes tendrían menos trabajo y se diligenciaría menos documentación si el sistema de gestión de la calidad no existiera. En cuanto a los docentes, la resistencia se genera en el trámite de la documentación para el sistema.

\section{Beneficios}

Los beneficios del sistema de gestión de la calidad certificado se han reconocido a lo largo de los seis años transcurridos desde su implementación y se concentran en el ajuste y la reducción de los procesos conforme al comportamiento y las necesidades del sistema de gestión de la calidad, el arraigamiento del pensamiento colectivo en términos de mejora continua, el ajuste de la documentación, la inmersión en el lenguaje de la calidad, el aporte al currículo y a la educación. Estos beneficios se destacan como relevantes y son producto del trabajo conjunto de líderes y miembros de las instituciones educativas.

La comunidad educativa en general reconoce que los beneficios también se ven reflejados en el mejoramiento de la infraestructura física y tecnológica, en las mejoras al plan de estudios y al currículo, la respuesta a las evaluaciones de los estudiantes en las pruebas nacionales, las mediciones y en la dinamización de los procesos, así como en la orientación del servicio hacia la satisfacción del estudiante.

Los beneficios establecidos como producto de la certificación de la calidad no solo permiten vislumbrar el cumplimiento de los requisitos que aseguran la conformidad con la NTC ISO 9001:2008, sino el avance paulatino de la cultura de la calidad. El total de los líderes coincide en el reconocimiento de beneficios, que se constituyen en un legado institucional y, por ende, de la calidad: la construcción colectiva de los procesos y los procedimientos acorde con los parámetros de contenido, siguiendo el ciclo de mejoramiento continuo PHVA, que aseguran la planificación, el seguimiento, la medición, el control y la integración desde lo humano; el establecimiento de mecanismos para la atención al cliente y la medición de su satisfacción; la construcción paulatina de una cultura de la calidad que involucra a los miembros de la comunidad educativa en el lenguaje del mejoramiento; y en la necesidad de cumplir con los requisitos de un sistema de gestión de la calidad certificado, la adecuación y construcción de infraestructura en concordancia con las necesidades de las instituciones, de los requisitos legales y de los requisitos de la comunidad educativa.

\section{Mejora proyectada}

Como resultado de la investigación también surgieronn estrategias de mejora continua para el direccionamiento estratégico, que conjugan los elementos de la gestión escolar con la gestión certificada en calidad 
bajo los parámetros de la NTC ISO 9001:2008. De este ejercicio surgieron las siguientes propuestas:

- El planteamiento de estrategias de mercadeo y publicidad para la captación de nuevos estudiantes, aprovechando la imagen positiva y el reconocimiento que se tiene de los colegios en la provincia.

- El establecimiento de convenios interinstitucionales para el fortalecimiento económico y el aprovechamiento de la infraestructura física y tecnológica existente, uno de los beneficios y factores de fortalecimiento del servicio educativo.

- El avance en la implementación de un sistema integrado de gestión entre el sistema de gestión de la calidad existente con el sistema de gestión ambiental bajo la norma técnica ISO 14001 y la norma técnica OHSAS 18001 para la gestión de la seguridad y la salud en el trabajo, que conjugue la madurez del sistema de gestión de la calidad y los resultados obtenidos a favor de la gestión institucional, con los elementos de los proyectos educativos transversales relacionados con el medio ambiente, el Proyecto Ambiental Escolar (PRAE), dado que su carácter transversal e interdisciplinario incorpora la visión sistémica del ambiente con la formación integral y los avances en materia de salud y seguridad del trabajador.

- El mantenimiento de las condiciones de mejoramiento e idoneidad de la infraestructura, de manera que se proteja la inversión económica, se refleje en la prestación del servicio educativo y en el favorecimiento de la calidad de la educación propia de los colegios de la provincia de Bogotá.

- El fortalecimiento de los procesos de enseñanzaaprendizaje, aprovechando para ello los avances que el sistema de gestión de la calidad brinda en cuanto a planificación académica, seguimiento y control, de manera que el avance en la cultura de la calidad y la preocupación por la conformidad con los requisitos no desvíe del objetivo máximo de la escuela, relacionado con la formación y el desarrollo de las competencias individuales y grupales.

- El establecimiento de estrategias de fortalecimiento de la conciencia de la calidad, de sus beneficios y de la importancia para el desarrollo de las actividades individuales y grupales, para así avanzar en la cultura de la calidad, especialmente en los colegios con puntuaciones en niveles bajo y muy bajo, como Centro, Duitama y Zipaquirá.

- El establecimiento de estrategias de fortalecimiento del liderazgo en las rectoras líderes de los procesos de la gestión directiva, con el fin de que adquieran elementos para la toma de decisiones, visión proyectiva y búsqueda de nuevas alternativas de manejo institucional que redunden en el aumento de los ingresos económicos, la conciencia de la calidad vista y proyectada desde la gerencia con una mirada superior al cumplimiento de requisitos básicos, el desarrollo de habilidades para la planificación estratégica a la vanguardia de la competencia y las necesidades del entorno, dispuestos a responder por los desafíos de la inclusión, la modernización y la globalización propuestos en la guía 34 del Ministerio de Educación Nacional.

- La dinamización del proceso de auditoría interna de calidad, de manera que el ejercicio de seguimiento permita el intercambio institucional de auditores internos o la contratación de auditores calificados, según la conveniencia de las instituciones.

- Finalmente, la revisión crítica de las prácticas, los procesos y resultados, a la luz de la identificación, la valoración y el tratamiento de los riesgos que se pueden presentar en la provincia, así como la construcción de un modelo de gestión del conocimiento 
a la luz de la pedagogía y la calidad, específicamente para cada colegio

Por otra parte, respecto al marco teórico, se encontró concordancia con los resultados obtenidos, ya que estos conjugan el alcance de la gestión en el ámbito escolar como un proceso sistemático orientado al fortalecimiento de las instituciones educativas y a sus proyectos, con el fin de enriquecer los procesos pedagógicos, directivos, comunitarios y administrativos (MEN, 2013) que frente al sistema de gestión de la calidad que desarrolla el ciclo PHVA -influenciado por los objetivos educativos, las definiciones del Proyecto Educativo Institucional, por los diversos métodos de enseñanza, la comunidad educativa y las prácticas administrativas y financieras de cada establecimiento- producen mejoramiento continuo y eficacia. Si retomamos los efectos en términos de lo que sigue por virtud de una causa (RAE, 2014, en línea), se analizaron los datos y establecieron las conclusiones con enfoque multidimensional, además, se aportaron las respuestas a las hipótesis y al problema planteado frente a la necesidad de la existencia de resultados medibles, tangibles y cuantificables.

La metodología desarrollada en el marco de un estudio explicativo, aplicado y mixto permitió intervenir en la realidad objeto de estudio y combinar el análisis de datos para la toma de decisiones con la interacción directa para el mejoramiento de los procesos estratégicos, misionales y de apoyo, acorde con los resultados obtenidos.

Así mismo, permitió comprobar las hipótesis planteadas frente a los efectos, los beneficios, la receptividad o el rechazo por parte de los líderes de los procesos y en lo administrativo.

\section{CONCLUSIONES}

Se logró el establecimiento de efectos positivos en cuanto a que la gestión de la calidad sirve de soporte para desarrollar los objetivos institucionales y aporta beneficios tangibles, como los siguientes: 1) la construcción colectiva de los procesos y los procedimientos, acorde con los parámetros de contenido y siguiendo el ciclo de mejoramiento continuo PHVA; 2) el establecimiento de mecanismos para la atención al cliente y la medición de su satisfacción; 3) la construcción paulatina de una cultura de la calidad que involucra a los miembros de la comunidad educativa en el lenguaje del mejoramiento y en la necesidad de cumplir con los requisitos de un sistema de gestión de la calidad certificado; 4) la adecuación y construcción de infraestructura en concordancia con las necesidades de las instituciones, de los requisitos legales y de los requisitos de la comunidad educativa.

En igual sentido, las hipótesis se corroboraron estableciendo que la certificación de la calidad ha contribuido y mejorado la calidad del servicio educativo y que es necesaria para el buen funcionamiento institucional. La investigación no encontró resultados que determinaran desgaste administrativo frente a los beneficios obtenidos.

Los resultados aportan a la ampliación del conocimiento frente a la conjugación de la educación y los sistemas de gestión de la calidad certificado y al enriquecimiento personal frente a la interacción con la comunidad educativa, ya que han servido de base para el desarrollo de mesas de actualización y análisis, así como para la toma de decisiones en torno a ajustes de carácter administrativo y misional. 


\section{REFERENCIAS}

Aristizábal, G. R., Martín, G., Cárdenas, R. y V. M. Buitrago. (2009). Impacto de la certificación ISO 9001:2000 en la visión y misión de una institución educativa de educación preescolar, básica y media certificada (Tesis de maestría). Pontificia Universidad Javeriana, Bogotá. Recuperado de goo.gl/klFyPr

Castañeda, E. (2010). El reto es consolidar el sistema de calidad educativa. Al Tablero, 56 [en línea]. Recuperado de goo.gl/aI6zXx

Instituto Colombiano de Normas Técnicas y Certificación - ICONTEC. (2011). Guía técnica colombiana GTC 137. Vocabulario. Bogotá: ICONTEC.

Medina, M. N. (2007). La investigación aplicada a proyectos: Identificación del proyecto y formulación de la investigación. Bogotá D.C: Ediciones Antropos.

Ministerio de Educación Nacional-MEN. (2016). Certificación y acreditación. Recuperado de http://www. mineducacion.gov.co/1621/w3-article-179263.

html . (2013).

Gestión educativa. Recuperado de http://www.mineducacion.gov.co/1621/w3-propertyvalue-48473. html

Organización de las Naciones Unidas para la Educación, la Ciencia y la Cultura - UNESCO. (2002). Declaración mundial sobre educación para todos y marco de acción para satisfacer las necesidades básicas de aprendizaje. Varsovia: UNESCO.

(2000).

Foro Mundial sobre la Educación. Marco de acción de Dakar. Francia: UNESCO. Recuperado de http:// unesdoc.unesco.org/images/0012/001211/121147s. pdf

Real Academia Española. (2014). Diccionario de la lengua española. Madrid: Espasa. 